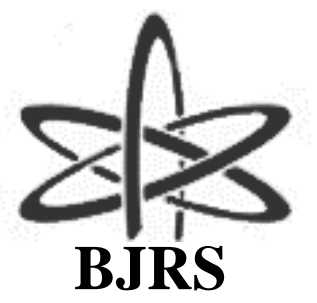

\author{
BRAZILIAN JOURNAL \\ $\mathrm{OF}$ \\ RADIATION SCIENCES \\ 07-1A (2019) 01-12
}

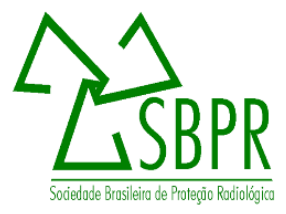

\title{
Análise dos índices de exposição de sistemas CR em exames de tórax
}

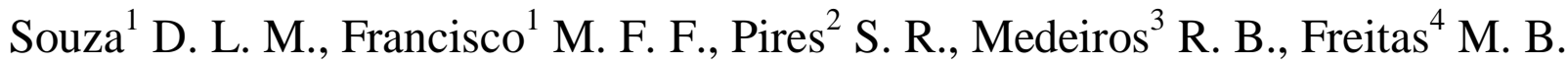 \\ ${ }^{1}$ Programa de Residência em Física Médica da Universidade Federal de São Paulo, São Paulo, Brasil \\ ${ }^{2}$ Departamento de Oncologia Clínica e Experimental da Escola Paulista de Medicina - \\ Universidade Federal de São Paulo, São Paulo, Brasil \\ ${ }^{3}$ Escola Paulista de Medicina - Universidade Federal de São Paulo, São Paulo, Brasil. \\ ${ }^{4}$ Departamento de Biofísica da Escola Paulista de Medicina - Universidade Federal de São Paulo, São Paulo, Brasil
}

dlmsouza90@gmail.com

\section{RESUMO}

O uso de sistemas de imagens digitais demandou a criação de padrões de exposição dos detectores empregados, particularmente em radiologia computadorizada (CR). O objetivo deste estudo foi avaliar índices de exposição empregados por um fabricante (S-Value - Fuji) e, mais recentemente, propostos pela IEC 62494-1 e AAPM Task Group 116 (índice de exposição padronizado e seu desvio - EI e DI). A avaliação foi realizada para exames de tórax em pacientes assistidos em dois hospitais públicos de grande porte. Para avaliar a dose de entrada na pele (DEP) dos pacientes examinados, foram coletadas as seguintes informações: tensão do tubo, produto correntetempo, distância foco-detector e espessura do paciente. Foram registrados os valores dos índices de exposição das imagens aceitas clinicamente. Em ambos hospitais constatou-se uma grande variação dos índices de exposição: $S$ Value de 419 a 4093 e DI de $-1,6$ a $+4,3$ (-30\% a +170\% em relação ao índice de exposição alvo $\left.-\mathbf{E I}_{\mathrm{T}}\right)$, indicando total desacordo com os valores recomendados. Esses resultados foram corroborados por meio da análise das DEP, alcançando em um dos hospitais valores em média 3,5 vezes acima dos níveis de referência recomendados. Verificou-se baixa correlação entre DEP e espessura dos pacientes, indicando a necessidade de um maior rigor na adequação das técnicas ao biótipo dos pacientes. Os resultados obtidos nos dois hospitais apontam para a necessidade de implementação de um programa de otimização das doses praticadas em associação com a qualidade da imagem e de treinamento para o uso adequado dos índices de exposição pelos técnicos.

Palavras-chave: proteção radiológica, diagnóstico por imagem, radiologia, medida de exposição à radiação, dose de radiação. 


\begin{abstract}
The use of digital imaging systems has required the creation exposure standards for detectors, particularly in computerized radiology (CR). The objective of this study was to evaluate the exposure index proposed by a manufacturer (S-Value - Fuji) and more recently by both IEC 62494-1 and AAPM Task Group 116 (standardized exposure index and their deviation - EI and DI). The evaluation was performed for chest examinations in patients assisted in two large public hospitals. The following information was collected to evaluate the entrance skin dose (ESD) of the patients examined: tube potential, current-time product, focusdetector distance, and patient thickness. Exposure index ( $S$-Value, EI and DI) from clinically accepted images were recorded. In both hospitals a large variation of the exposure index was observed: S-Value from 419 to 4093 and DI from -1.6 to $+4.3(-30 \%$ to $+170 \%$ in relation to the target exposure index - EIT), indicating total disagreement with the recommended values. These outcomes were corroborated by the ESD analysis, reaching in one of the hospitals values on average 3.5 times above the recommended reference levels. Low correlation between DEP and patient thickness was noticed, indicating the need for a greater accuracy in the adequacy of the techniques to the patients' body type. The results obtained in the two hospitals point that a program of optimisation of doses practiced in association with image quality must be implemented and training for the adequate use of exposure index by thechnologists is required.
\end{abstract}

Keywords: radiation protection, diagnostic imaging, radiology, radiation exposure measurement, radiation dose.

\title{
1. INTRODUÇÃO
}

A evolução da radiologia, passando de sistemas analógicos (tela-filme) para sistemas digitais (CR e DR), proporcionou melhor eficiência na produção de imagens com qualidade clínica, criando a falsa sensação que os novos sistemas não estão sujeitos a falhas na seleção dos parâmetros técnicos (tensão, produto corrente-tempo, distância foco-filme, etc). No sistema tela-filme o próprio detector fornece um indicativo da dose recebida pelo paciente na medida em que o contraste da imagem é alterado diretamente pela seleção dos parâmetros técnicos empregados na realização do exame. Já nos detectores digitais, a ampla faixa dinâmica de resposta à exposição permite super ou subexposições com pouca ou nenhuma influência no contraste da imagem, praticamente eliminando a relação existente nos sistemas tela-filme entre dose no paciente e qualidade da imagem radiológica. O processamento de contraste nos sistemas digitais está associado ao ajuste automático da escala de tons de cinza na imagem, considerando um nível desejável de visualização das 
estruturas. A perda dessa relação associada em muitos casos à falta de conhecimento dos novos parâmetros empregados nos sistemas digitais, dentre esses, os índices de exposição (EI), tem produzido uma lacuna na capacidade de realizar garantia e melhoria da qualidade nesses sistemas. O emprego e interpretação adequados dos índices de exposição em radiografia digital são de fundamental importância para garantir imagens radiológicas com qualidade clínica e para evitar que os pacientes recebem dose de radiação desnecessária [1]. Soma-se a isso, o fato que até recentemente os fabricantes empregavam diferentes nomenclaturas para definir a exposição à radiação em seus detectores de imagens digitais (diferentes índices de exposição [2]), dificultando a sua interpretação na prática diária, o compartilhamento de dados entre as instituições e a criação de bancos de dados de registro de doses de pacientes. Esse aspecto foi melhorado recentemente pela implementação de um padrão internacional que define um único índice de exposição para todos os sistemas de imagem digitais em radiologia $[3,4]$.

Diferentemente dos índices de exposição propostos pelos fabricantes, esse novo índice padronizado permite uma determinação direta do kerma no ar no receptor de imagem $\left(\mathrm{K}_{\mathrm{ar}}\right)$ em sistemas CR ou DR, que varia linearmente com o produto corrente-tempo (mAs) empregado, para uma tensão fixa $(\mathrm{kVp})$, na faixa dinâmica de resposta do detector:

$$
\mathrm{EI}=100\left(\mu \mathrm{Gy}^{-1}\right) \times \mathrm{K}_{\mathrm{ar}}(\mu \mathrm{Gy})
$$

Para facilitar sua interpretação na prática clínica, também foi definido o desvio (DI) desse índice de exposição em relação a um índice de exposição alvo $\left(\mathrm{EI}_{\mathrm{T}}\right)$ previamente definido[4]:

$$
\mathrm{EI}=10 \times \log _{10} \frac{\mathrm{EI}}{\mathrm{EI}_{\mathrm{T}}}
$$

$\mathrm{O} \mathrm{EI}_{\mathrm{T}}$ é a referência de exposição obtida quando o detector é exposto de forma ótima, considerando a razão sinal-ruído no detector que se deseja obter para uma dada região anatômica examinada na imagem radiológica. Este índice de exposição alvo pode ser sugerido pelo fabricante do sistema de imagem digital, mas deve ser obtido a partir das necessidades diagnósticas da imagem 
radiológica de interesse, sendo normalmente estabelecido em processos de otimização [5,6,7,8]. Considerando a escala logarítmica que relaciona DI e a razão dos EIs, observa-se que um exame radiológico praticado com um $\mathrm{EI}$ duas vezes superior ao $\mathrm{EI}_{\mathrm{T}}$ resulta numa diferença do DI de +3 , enquanto uma diferença de -3 representa que o IE praticado é metade do valor do $\mathrm{EI}_{\mathrm{T}}$.

Pelo exposto, este estudo teve como objetivo analisar o impacto prático do uso de diferentes índices de exposição associados a detectores digitais do tipo Image Plate (IP) empregados na radiologia computadorizada (CR): $S$-Value proposto pelo fabricante Fujifilm[2] e o IE padronizado proposto pela IEC-AAPM[3,4], na dose de radiação recebida por pacientes submetidos a exames de tórax.

\section{MATERIAS E MÉTODOS}

O estudo foi conduzido nos setores de radiologia de dois hospitais gerais públicos de grande porte localizados na região metropolitana de São Paulo - Brasil, responsáveis pela realização de aproximadamente 220 e 60 mil exames radiológicos anualmente, respectivamente, atendendo pacientes provenientes de serviços ambulatoriais de diversas especialidades. Considerando que em ambos os hospitais os exames de tórax representam aproximadamente $40 \%$ do total de exames realizados, o estudo foi dirigido a este exame, nas respectivas salas onde se concentravam a maior parte dos exames dessa modalidade. Ambos os hospitais possuíam equipamentos de raios $\mathrm{X}$ trifásicos (Emerix 50 - Medicor e XR 6000-General Electric, respectivamente) com sistemas de imagem do tipo CR: Agfa - Modelo CR 85-X e Fuji - Modelo FCR - Profect CS Plus, sendo que um deles já operava com o novo EI proposto pela IEC-AAPM e outro ainda operava com a definição anterior de índice de exposição fornecida pelo fabricante ( $S$-Value - Fujifilm). Uma amostra de 46 exames de tórax (projeção póstero-anterior - PA) foi analisada, considerando o registro, no momento de realização do exame, da espessura do paciente (biótipo) e dos parâmetros técnicos empregados na sua realização: tensão $(\mathrm{kVp})$, produto corrente-tempo (mAs) e distância foco-pele (dfp). Estas informações, juntamente com os valores de rendimento ( $\mathrm{R}$ - mGy/mAs @ 1m para valores diversos de $\mathrm{kVp}$ ) dos equipamentos radiológicos empregados na realização dos exames 
e do fator de retroespalhamento típico (FBS) para feixes com qualidade semelhante[9], permitiram a determinação da dose de entrada no paciente (DEP):

$$
\mathrm{DEP}=\frac{\left[\mathrm{FBS} \times \mathrm{R}\left(\mathrm{a} \times \mathrm{kVp} \mathrm{p}^{2}+\mathrm{b} \times \mathrm{kVp}+\mathrm{c}\right) \times \mathrm{mAs}\right]}{(\mathrm{dfp})^{2}},
$$

onde $a, b$ e $c$ são parâmetros (constantes) determinados pelo ajuste de uma função polinomial de $2^{\circ}$ grau que relaciona o rendimento @ $1 \mathrm{~m}$ para diversos valores de $\mathrm{kVp}$. Os valores de rendimento foram determinados durante a realização dos testes de controle de qualidade, quando também se verificou eventuais diferenças entre os valores nominais e reais de $\mathrm{kVp}$ e mAs em toda faixa de operação dos equipamentos.

Além dos parâmetros associados ao paciente e ao protocolo empregado, também foram registrados os valores associados aos índices de exposição dos sistemas CR empregados: S-Value, índice de exposição (EI), índice de exposição alvo $\left(\mathrm{EI}_{\mathrm{T}}\right)$ e desvio do índice de exposição (DI). Como valores de $\mathrm{EI}_{\mathrm{T}}$ foram empregados os valores propostos pelo fabricante (Agfa), quando da instalação do sistema $\mathrm{CR}\left(\mathrm{EI}_{\mathrm{T}}=450\right)$. Somente foram registrados os valores dos índices de exposição com colimação física adequada no momento de realização do exame, não tendo sido empregada, portanto, qualquer tipo de colimação eletrônica no processamento da imagem. Este procedimento evitou que valores dos índices de exposição "falsos" fossem empregados na análise realizada neste estudo. Todos os exames foram realizados empregando IPs de tamanho $35 \mathrm{x} 43 \mathrm{~cm}$. Todas as imagens apresentaram qualidade diagnóstica, ou seja, o técnico responsável pela realização da radiografia considerou que a imagem produzida do tórax do paciente atendia à justificativa clínica (hipótese diagnóstica) para a solicitação do exame.

Enquanto o IE se relaciona diretamente com a energia absorvida do detector, conforme definido pela IEC-AAPM[3,4], o S-Value, ou Sensitivity, adotado pelo fabricante Fujifilm, tem comportamento inversamente proporcional à exposição no detector[2]:

$$
\mathrm{S} \approx \frac{200}{\mathrm{X}(\mathrm{mR})}
$$


Esse comportamento inverso muitas vezes pode gerar erros na sua interpretação, dificultando seu emprego rotineiro na prática clínica. Por outro lado, o novo índice de exposição EI e seu desvio em relação ao $\mathrm{EI}_{\mathrm{T}}$ (DI) supostamente permitem uma interpretação mais objetiva, conforme recomendado no Report 116 da AAPM[4] (Tabela 1).

Tabela 1: Faixa principal de variação de DI e as respectivas interpretações (adaptado de AAPM Report 116[4]).

\begin{tabular}{|c|c|}
\hline DI & Interpretação do DI \\
\hline \multirow{2}{*}{$<-3,0$} & Subexposição \\
\hline & Repetir \\
\hline \multirow{2}{*}{$-3,0$} & Subexposição \\
\hline & Consultar radiologista para repetir \\
\hline \multirow{2}{*}{$-1,0$} & Subexposição \\
\hline & Consultar radiologista para repetir \\
\hline$-0,5$ & Faixa ótima \\
\hline$+0,5$ & Faixa ótima \\
\hline \multirow{2}{*}{$+1,0$} & Superexposição \\
\hline & Somente repetir a imagem se a anatomia não puder ser visualizada \\
\hline \multirow{2}{*}{$+3,0$} & Superexposição \\
\hline & Somente repetir a imagem se a anatomia não puder ser visualizada \\
\hline \multirow{2}{*}{$>+3,0 *$} & Dose excessiva ao paciente \\
\hline & Somente repetir se a anatomia não puder ser visualizada \\
\hline
\end{tabular}

$\mathrm{Na}$ análise dos diferentes índices de exposição dos sistemas CR empregados neste estudo também foi avaliado como os técnicos responsáveis pela realização dos exames interpretavam os resultados obtidos e que procedimentos ou comentários eram feitos a partir da informação do valor do respectivo índice de exposição obtido no momento da leitura do IP. 


\section{RESULTADOS E DISCUSSÕES}

Os dados obtidos do biotipo dos pacientes e dos parâmetros técnicos empregados nos exames de tórax em cada um dos hospitais participantes do estudo, com o respectivo tamanho da amostra e tipo de sistema CR e índice de exposição empregado, pode ser observado na Tabela 2.

Tabela 2: Tamanho da amostra, estatística descritiva dos dados coletados e sistemas CR empregados nos dois hospitais participantes do estudo.

\begin{tabular}{|c|c|c|c|c|c|}
\hline $\begin{array}{l}\text { Instituição } \\
\text { (tamanho da } \\
\text { amostra) }\end{array}$ & $\begin{array}{c}\text { Sistema CR } \\
\text { (índice de } \\
\text { exposição) }\end{array}$ & $\begin{array}{c}\text { Espessura } \\
\text { do paciente } \\
\text { (cm) } \\
\text { (máx-mín) }\end{array}$ & $\begin{array}{c}\text { Tensão } \\
\left(\mathbf{k V} \mathbf{V}_{\mathbf{p}}\right) \\
\text { (máx-mín) }\end{array}$ & $\begin{array}{c}\text { Produto } \\
\text { corrente-tempo } \\
(\mathbf{m A s}) \\
\text { (máx-mín) }\end{array}$ & $\begin{array}{c}\text { Distância } \\
\text { foco-pele } \\
\text { (cm) } \\
\text { (máx-mín) }\end{array}$ \\
\hline $\begin{array}{l}\text { Hospital A } \\
(\mathrm{N}=18)\end{array}$ & $\begin{array}{c}\text { Agfa } \\
\left.\text { (IE padrão }^{(3,4)}\right)\end{array}$ & $\begin{array}{l}24,6 \pm 2,4 \\
(29-21)\end{array}$ & $\begin{array}{l}116,5 \pm 1,8 \\
(117-109)\end{array}$ & $\begin{array}{c}14,3 \pm 3,5 \\
(20-10)\end{array}$ & $\begin{array}{c}160 \pm 15 \\
(185-118)\end{array}$ \\
\hline $\begin{array}{l}\text { Hospital B } \\
(\mathrm{N}=28)\end{array}$ & $\begin{array}{c}\text { Fujifilm } \\
(\text { S-Value } \\
(\mathbf{2})\end{array}$ & $\begin{array}{c}28,0 \pm 4,9 \\
(37-15)\end{array}$ & $\begin{array}{l}88,1 \pm 7,6 \\
(114-78)\end{array}$ & $\begin{array}{c}6,3 \pm 4,4 \\
(25-4)\end{array}$ & $\begin{array}{l}167,5 \pm 9,9 \\
(191-148)\end{array}$ \\
\hline
\end{tabular}

A Figura 1 apresenta os valores de DEP em termos da espessuras dos pacientes examinados em ambos os hospitais.

Figura 1: Doses de entrada na pele (DEP) em função das espessuras dos pacientes submetidos a radiografias de tórax nos dois ospitais (Hospital A e Hospital B).

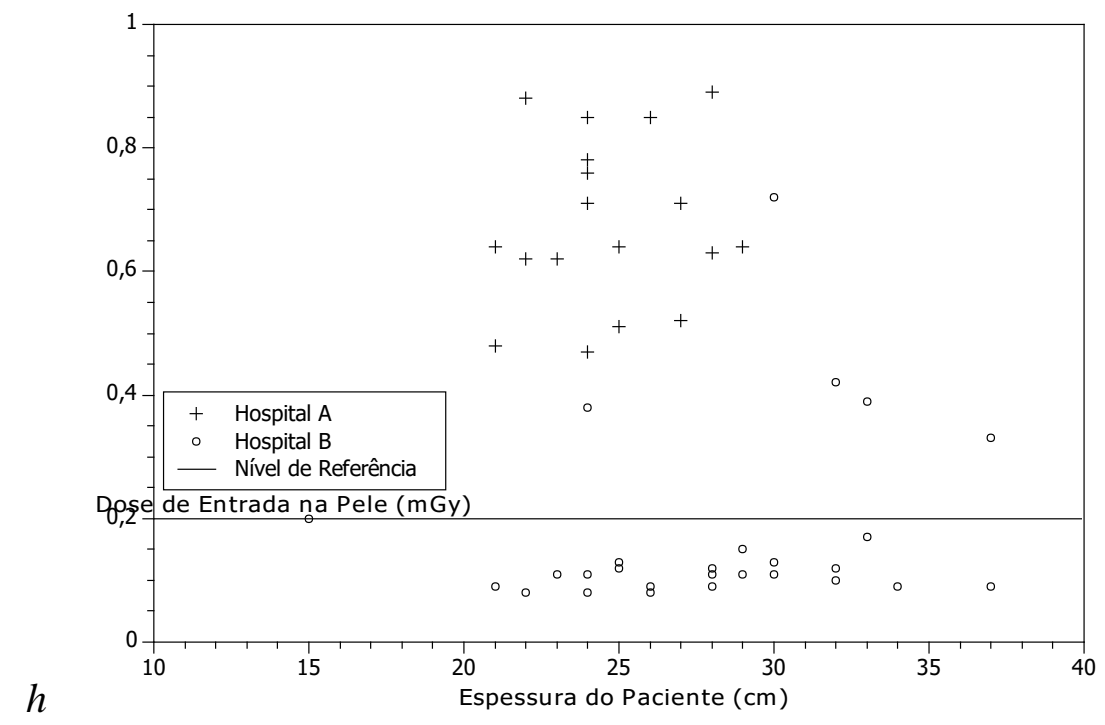


Observa-se claramamente que em ambos hospitais as DEPs obtidas praticamente não guardam relação com a espessura do paciente, em desacordo com as recomendações[10], conforme demonstram os coeficientes de correlação calculados para ambos conjuntos de dados $\left(\mathrm{R}^{2}<0,1\right)$. Verifica-se um comportamento inverso das DEPs praticadas nos hospitais em relação ao nível de referência (NR) recomendado para um paciente adulto pela legislação brasileira[10] para exames de tórax PA (0,2mGy). Enquanto que em um deles os valores de DEP estão em média 340\% acima do NR, no outro observa-se DEPs em média 14\% abaixo do NR recomendado. A análise da distribuição dos parâmetros técnicos empregados $\left(k V_{P}, m A s\right.$, dfp) na realização dos exames em cada um dos hospitais (Tabela 2) reforça os valores de DEP obtidos, indicando a necessidade de adequação das técnicas as características físicas dos pacientes (biótipo).

A Figura 2 apresenta a distribuição percentual dos valores de DI (Figura 2a) e S-Value (Figura 2b) obtidos nos sistemas CR.

Figura 2: Distribuição percentual dos índices de exposição praticados em cada um dos hospitais:

(a) Hospital A - DI e (b) Hospital B - S-Value.
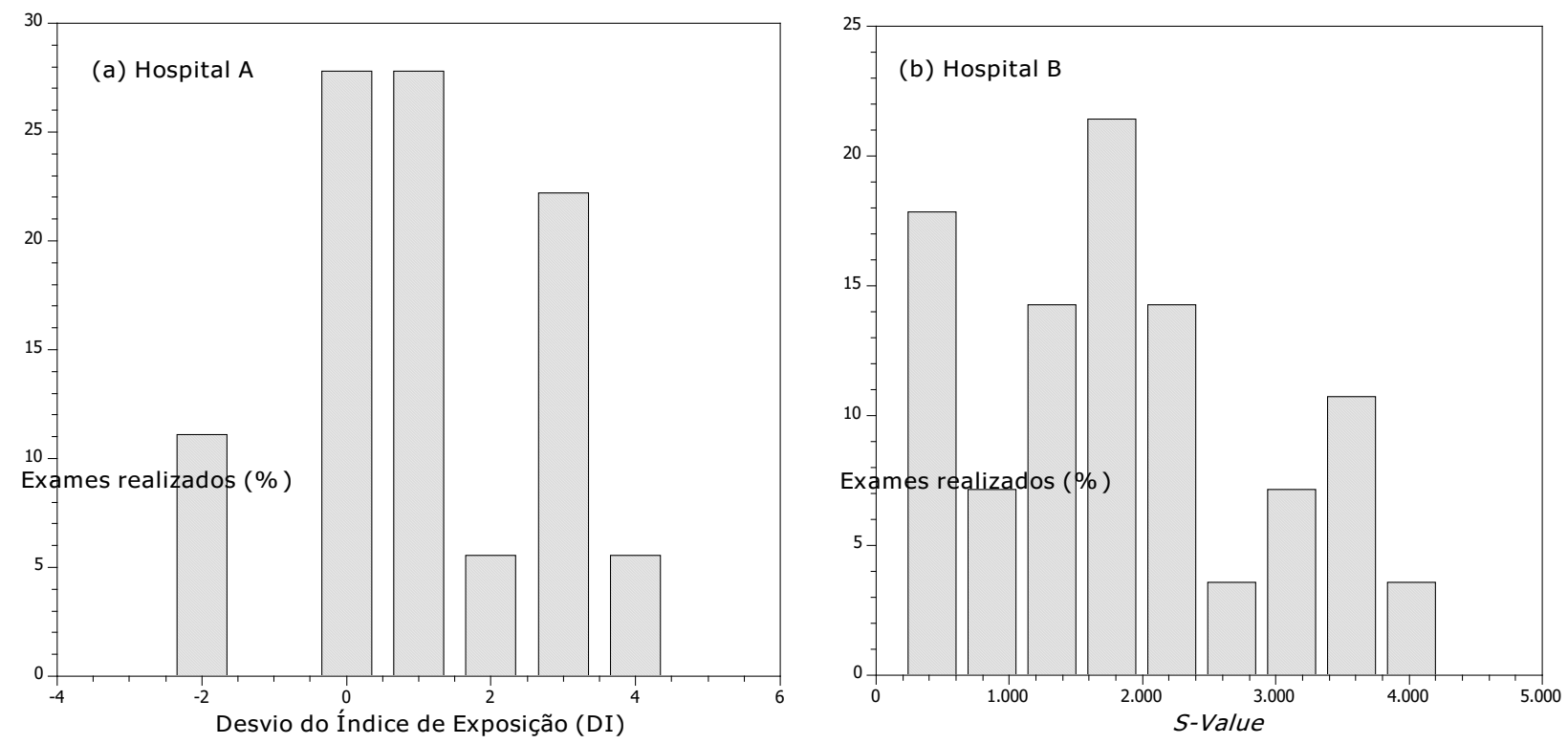
A análise dos valores de DI (Hospital A) demostra uma variação de -1,6 até +4,3, o que representa uma diferença relativa de $-30 \%$ a $+170 \%$ do $\mathrm{EI}$ em função do $\mathrm{EI}_{\mathrm{T}}$ recomendado pelo fabricante $\left(\mathrm{EI}_{\mathrm{T}}=450\right)$. Essa distribuição revela que aproximadamente $60 \%$ dos exames estão sendo praticados com superexposição do detector, sendo que aproximadamente $25 \%$ apresentam DI $\geq+3$ (dobro do $\mathrm{EI}_{\mathrm{T}}$ ) e que $100 \%$ apontam para doses de radiação excessiva no paciente, conforme observa-se na Figura 1. Observa-se que somente $28 \%$ dos exames foram realizados na faixa ótima de exposição do detector (Tabela 1). Esses aspectos reforçam novamente a necessidade de otimização das técnicas que considerem a variação do biótipo do paciente.

Considerando que esse sistema CR havia acabado de ser instalado no setor, verifica-se que mesmo com a nova definição do índice de exposição e seu desvio, sua correta interpretação não foi realizada na prática clínica[4]. A substituição dos IPs foi relatada pelos técnicos como um agravante para os índices de exposição praticados, considerando que os parâmetros técnicos empregados na realização dos exames não foram adequadamente corrigidos para a resposta de detecção dos novos IPs, ou seja, não foi implementado um processo de otimização no setor após a instalação do novo sistema CR. Além desse fato, os resultados indicam uma tendência de emprego de imagens com baixo ruído, sugerindo o efeito dose creep[6]. Verificou-se como esperado que a colimação eletrônica alterava o índice de exposição (EI) e, portanto, o DI.

Por outro lado, a análise da distribuição dos valores de $S$-Value (Hospital B) aponta para condições de subexposição do detector, sendo que somente $18 \%$ dos exames coletados foram praticados com índice de exposição na faixa recomendada (200 a 800)[11]. Vale destacar que os IPs deste setor de radiologia já possuíam um tempo de uso elevado, o que poderia justificar a baixa sensibilidade dos detectores e, possivelmente, índices de exposição que não satisfazem os padrões de desempenho (calibração). No Brasil ainda não é exigido o controle de qualidade de sistemas de imagem digital[10]. Estes resultados reforçam novamente a necessidade de implementação de um programa de otimização da qualidade da imagem radiológica em associação com as doses praticadas nos pacientes, considerando também a avaliação da qualidade dos IPs.

A variação da DEP com os índices de exposição praticados pode ser observado na Figura 3. Como esperado não se observou nenhuma correlação significativa com os índices de exposição, embora em ambos os casos os valores de DI e $S$-Value encontrados, indiquem a necessidade de 
otimização das práticas empregadas na realização dos exames de tórax pelos setores responsáveis de cada hospital.

Se em um dos hospitais verifica-se a necessidade de redução das DEPs (Hospital A), confirmada pela análise dos valores de DI (Figura 2a), no outro (Hospital B) a situação é inversa: os valores dos índices de exposição $S$-Value praticados (Figura 2b) sugerem que a qualidade das imagens pode ser melhorada aumentando-se a razão sinal-ruído nos IPs e, consequentemente, a exposição à radiação do detector. Em ambos os casos, há necessidade de implementar um programa de otimização que considere tanto os índices de exposição dos sistemas de imagem como os indicadores de dose de radiação (DEP) praticados nos pacientes, incluindo a avaliação da qualidade diagnóstica da imagem pela equipe médica.

Figura 3. Variação da dose de entrada na pele (DEP) com o desvio do índice de exposição no IP (DI) e o S-Value.

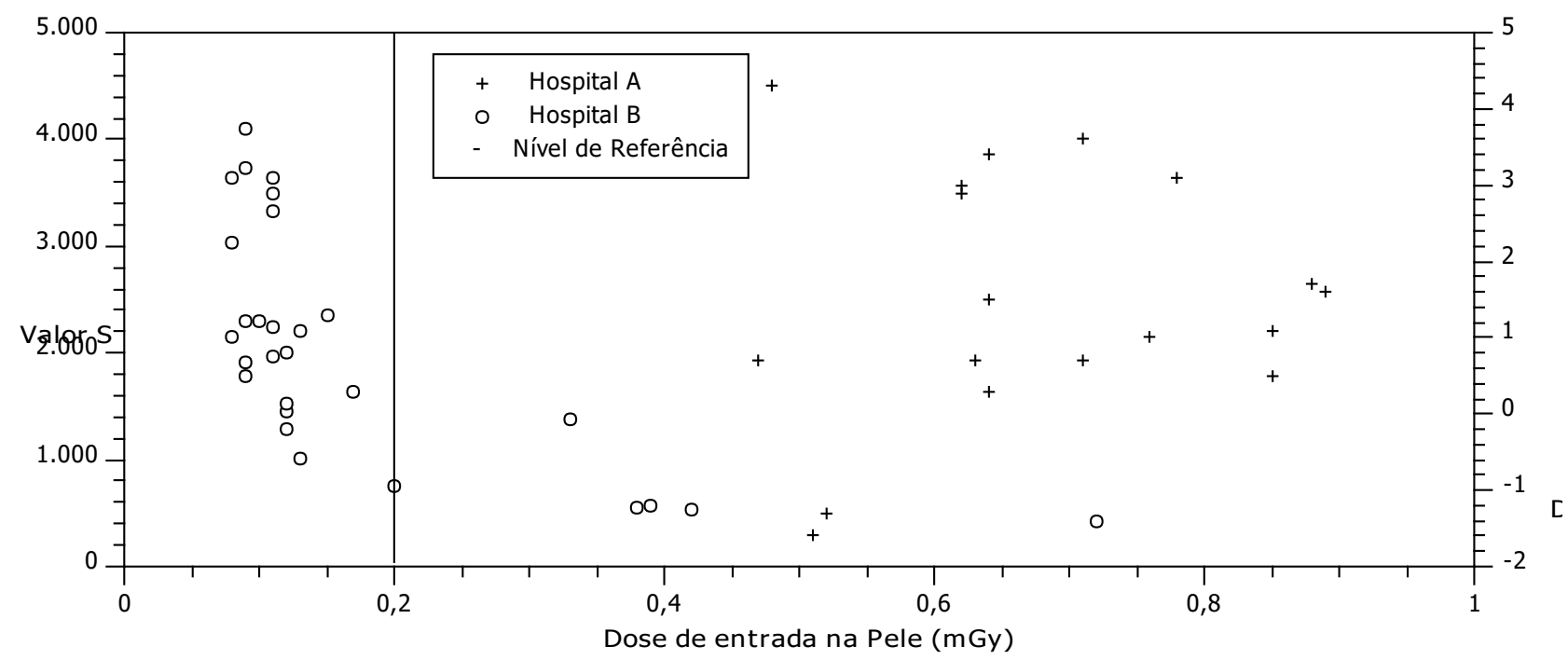

\section{CONCLUSÕES}

$\mathrm{O}$ advento dos sistemas de imagem digital com detectores que respondem numa ampla faixa de exposições pode levar ao uso de parâmetros técnicos $\left(\mathrm{kV}_{\mathrm{p}}, \mathrm{mAs}\right.$, distância foco-pele, etc.) que não estão associados ao biótipo do paciente. Essa prática pode resultar em doses de entrada na pele 
muito acima do níveis de referência recomendados ou em imagens com baixa qualidade (alto nível de ruído).

Embora a padronização dos índices de exposição seja de extrema importância para a prática dos setores de radiologia, a sua simples implementação não garante a correta interpretação em cada exame. Mesmo no setor onde já se encontrava instalado o software de padronização, é evidente a falta de conhecimento dos envolvidos no trabalho sobre como empregá-lo na melhoria das práticas. Esses resultados, associados aos valores praticados de DEP, apontam para a necessidade de otimização de técnicas de aquisição empregadas nos exames de tórax e treinamento da equipe técnica do setor em termos da interpretação dos índices de exposição.

A análise dos índices de exposição em associação com as DEPs revelou-se numa estratégia importante para o controle dos níveis de exposição à radiação dos pacientes e dos detectores empregados nos sistemas CR, independente da nomenclatura do índice de exposição empregado.

Resumindo, fica claro que a padronização dos índices de exposição (EI), embora importante para otimização dos sistemas de imagem digital, não pode estar dissociado de uma forte capacitação da equipe em termos das novas tecnologias, dos processos de otimização e dos aspectos de proteção radiológica nas exposições médicas.

\section{AGRADECIMENTOS}

Os autores agradecem às equipes dos setores de radiologia dos hospitais participantes e ao suporte financeiro do Ministério da Educação para as bolsas dos residentes do Programa de Residência em Física Médica da Universidade Federal de São Paulo.

\section{REFERÊNCIAS}

[1] WILLIAMS, M. B. Digital radiography image quality: Image acquisition. J. Amer. College Radiol., v. 4, p. 371-388, 2007. 
[2] AAPM - American Association of Physicists in Medicine. Acceptance Testing and Quality Control of Photostimulable Storage Phosphor Imaging Systems. AAPM Report 93. Task Group 10, 2006

[3] IEC - International Electrotechnical Commission - 62494-1 Ed. 1: Medical electrical equipment - Exposure index of digital x-ray imaging systems - Part 1: Definitions and requirements for general radiography, 2008.

[4] AAPM - American Association of Physicists in Medicine - An exposure indicator for digital radiography. AAPM Report 116. Task Group 116, Vol. 36, 2009.

[5] MATTSSON E. Optimization strategies in medical x-ray imaging. Radiat Prot Dosimetry, v. 114, p. 1-3, 2015.

[6] SEERAM E; DAVIDSON R; BUSHONG S; SWAN H. Radiation dose optimization research: exposure technique approaches in CR imaging e a literature review. Radiography, v. 19(4) p. 331-8, 2013.

[7] SEIBERT J. A; MORIN R. L; The standardized exposure index for digital radiography: an opportunity for optimization of radiation dose to the pediatric population. Pediatric Radiology, v. 41(5) p. 573-581, 2011.

[8] WILLIS C. E; Optimizing digital radiography of children. Eur J Radiol., v. 72(2), p. 266-73, 2009.

[9] ICRU - International Commission on Radiation Units and Measurements. Patient Dosimetry for X Ray used in Medical Imaging. ICRU Report 74. ICRU, 2005.

[10] Brasil. Ministério da Saúde. Agência Nacional de Vigilância Sanitária. Portaria $n^{\circ} 453$, de 01 de junho de 1998, Aprova o Regulamento Técnico que estabelece as Diretrizes Básicas de Proteção Radiológica em Radiodiagnóstico Médico e Odontológico. Diário oficial [da Republica Federativa do Brasil]. Brasília, 1998

[11] SEERAM, E. The New Exposure Indicator for Digital Radiography. J Med Imaging Radiat Sci 45, 144-158, 2014. 